\title{
Using Generative Adversarial Networks to Optimise Health Outcomes of Cities by Transforming Urban Design
}

\author{
Jasper S. Wijnands ${ }^{1}$, Kerry A. Nice ${ }^{1}$, Jason Thompson ${ }^{1}$, Haifeng Zhao ${ }^{1}$, Mark Stevenson ${ }^{1,2,3}$ \\ ${ }^{1}$ Transport, Health and Urban Design, Melbourne School of Design, the University of Melbourne \\ Grattan St, Parkville, Australia \\ jasper.wijnands@unimelb.edu.au; kerry.nice@unimelb.edu.au; jason.thompson@unimelb.edu.au; \\ haifeng.zhao@unimelb.edu.au; mark.stevenson@unimelb.edu.au \\ ${ }^{2}$ Melbourne School of Engineering, the University of Melbourne \\ Grattan St, Parkville, Australia \\ ${ }^{3}$ Melbourne School of Population and Global Health, the University of Melbourne \\ Grattan St, Parkville, Australia
}

\section{Extended Abstract}

Deep learning using neural networks has provided advancements in image style transfer, which merges the style of one image (e.g., a painting) and the content of another image (e.g., a photo). Our research shows this concept can be extended to improve urban design practices. Specifically, this study proposes an innovative computer-generated urban design methodology to facilitate goal-oriented amendments to the urban environment. Whereas traditional design generally focusses on designing small, local areas (i.e., a bottom-up approach), this new method can cover large areas using observation-based design. Our research has two main objectives; (i) to present a new technique for urban design based on big data and artificial intelligence; and (ii) to present findings of associations between health outcomes and specific urban design elements discovered using this methodology.

Methodology. A Victorian survey [1] among 34,000 people was used to identify the spatial distribution of health and wellbeing outcomes, including general health, feeling safe at night and social capital. For each outcome, the ten percent best and worst locations were grouped into two sets (i.e., domains). Google Street View (GSV) images from each location sampled urban design from an observer's point of view, leading to over 80,000 GSV images per domain. Since no matched pairs of images exist, supervised learning is not feasible. However, generative adversarial networks (GANs) have proven to be successful for style transfer using unsupervised learning. GANs consist of multiple competing models, namely generator (generating images in the new style) and discriminator (assessing if the generated image looks realistic) neural networks. Our approach is based on unsupervised image-to-image translation [2] and consists of two variational autoencoders [3] plus two discriminator models, calibrated for 1.2 million iterations using GPU computing. GSV images were then translated from one domain to the other, preserving the main structure of the input image (cf. 'content'), but transforming the 'style' from bad to good health outcomes (for example). The method is flexible with respect to a designer's aims, as the target domain can represent a mix of desired outcomes. Finally, the method has potential for immersive virtual reality applications by mass translating images, showing shifts in design style in a cost-effective manner, without the need for a (slower, more expensive) bottom-up urban design approach.

Findings. The translation from good to bad general health showed that buildings appearing close to the GSV camera were moved further away, indicating compactness of the urban environment is associated with good general health. This is consistent with [4], who found compact city design yields health gains of 420-826 disability-adjusted life-years per 100,000 population. Further observations include the positive impact of green space (e.g., grass, bushes and trees) on general health. The translation of areas where people feel unsafe at night to a safe style yielded taller trees. This is supported by [5], who found a significant positive correlation between the perceived safety of GSV images and visibility of vegetation higher than $2.5 \mathrm{~m}$. For the reverse translation, footpaths were removed, multi-level buildings reduced to single-level and more open space was created, indicating that people feel safer when not exposed to walking outside at night in low-density, open areas. An increase in social capital is associated with more grass, new or wider footpaths, smaller trees and the removal of fences. We speculate that reduced privacy measures (i.e., fences, large trees) and suitable outdoor space and infrastructure (i.e., grass, 
footpaths) support social interaction. Quantitative analyses using all domain images computed the 'average' translation by the generator neural network. This provided an insight into the network's internals and confirmed the findings above. Overall, our research provides theorists and researchers a method to investigate key features associated with the perception and impact of urban design and provides urban designers an alternative, empirical design methodology.

\section{References}

[1] Department of Health and Human Services, "Victorian Population Health Survey 2015: Selected survey findings," State of Victoria, Melbourne, 2017

[2] M. Y. Liu, T. Breuel and J. Kautz, "Unsupervised image-to-image translation networks," in Advances in Neural Information Processing Systems, Barcelona, vol. 30, pp. 700-708, 2016.

[3] D. P. Kingma and M. Welling, "Auto-encoding variational Bayes," arXiv preprint, arXiv:1312.6114, 2013.

[4] M. Stevenson, J. Thompson, T. H. de Sá, R. Ewing, D. Mohan, R. McClure, I. Roberts, G. Tiwari, B. Giles-Corti, X. Sun, M. Wallace and James Woodcock, "Land use, transport, and population health: estimating the health benefits of compact cities," The Lancet, vol. 388, no. 10062, pp. 2925-2935, 2016.

[5] X. Li, C. Zhang and W. Li, "Does the Visibility of Greenery Increase Perceived Safety in Urban Areas? Evidence from the Place Pulse 1.0 Dataset," ISPRS Int. J. Geo-Inf, vol. 4, no. 3, pp. 1166-1183, 2015. 